\title{
Fibroblast growth factor receptor 1 amplification is a common event in squamous cell carcinoma of the head and neck
}

Friederike Göke ${ }^{1,2,7}$, Maike Bode ${ }^{1,2,7}$, Alina Franzen ${ }^{1,2}$, Robert Kirsten ${ }^{2}$, Diane Goltz ${ }^{1}$, Antonia Göke ${ }^{2}$, Rakesh Sharma ${ }^{2}$, Diana Boehm ${ }^{2}$, Wenzel Vogel ${ }^{2}$, Patrick Wagner ${ }^{3}$, Claudia Lengerke $^{4}$, Glen Kristiansen ${ }^{1}$, Jutta Kirfel ${ }^{1}$, Tobias Van Bremen ${ }^{5}$, Friedrich Bootz ${ }^{5}$, Lynn E Heasley ${ }^{6}$, Andreas Schröck ${ }^{2,5,7}$ and Sven Perner ${ }^{1,2,7}$

${ }^{1}$ Institute of Pathology, University of Bonn, Bonn, Germany; ${ }^{2}$ Department of Prostate Cancer Research, University of Bonn, Bonn, Germany; ${ }^{3}$ Winchester Medical Center, Winchester, VA, USA; ${ }^{4}$ Department of Internal Medicine II, University of Tubingen, Tubingen, Germany; ${ }^{5}$ Department of Otorhinolaryngology/Head and Neck Surgery, University of Bonn, Bonn, Germany and ${ }^{6}$ Department of Craniofacial Biology, School of Dental Medicine, University of Colorado Anschutz Medical Campus, Aurora, CO, USA

\begin{abstract}
Recently, we characterized fibroblast growth factor receptor 1 amplification as a target for a rational therapy in lung squamous cell carcinoma. Patients harboring this genetic event are currently eligible for treatment with antifibroblast growth factor receptor small-molecule inhibitors in phase I clinical trials. This has the potential to significantly improve standard therapy for lung squamous cell carcinoma patients. The aim of this study was to elucidate whether fibroblast growth factor receptor 1 amplification is also a common genetic event in head and neck squamous cell carcinoma. For this purpose, we assembled a cohort of 555 patients, including 264 with metastatic disease and 147 with recurrent disease. Formalin-fixed, paraffin-embedded material of primary tumors, metastases and recurrences were assessed for fibroblast growth factor receptor 1 copy number status using fluorescence in situ hybridization. Human papilloma virus status was detected by p16 immunohistochemistry staining and PCR-ELISA. Molecular parameters were correlated with each other and with clinicopathological data. We found $15 \%$ of primary head and neck squamous cell carcinoma to display a fibroblast growth factor receptor 1 amplification. In nearly all cases, metastatic and recurrent tumor samples shared the same amplification status as the corresponding primary tumor. Fibroblast growth factor receptor 1 amplification was associated with nicotine and alcohol consumption, but was mutually exclusive with human papilloma virus infection. Amplification of the gene was associated with parameters of worse outcome. Our data identify fibroblast growth factor receptor 1 amplification as a frequent event in primary and metastatic head and neck squamous cell carcinoma and represents a potential biomarker for more aggressive disease. Fibroblast growth factor receptor 1-amplified tumors could potentially comprise a subset of head and neck squamous cell carcinoma against which targeted small-molecule inhibitors hold therapeutic efficacy. Modern Pathology (2013) 26, 1298-1306; doi:10.1038/modpathol.2013.58; published online 26 April 2013
\end{abstract}

Keywords: amplification; biomarker; fibroblast growth factor receptor 1; head and neck squamous cell carcinoma; HPV

Molecular profiling of human malignancies has become a major focus of research over the past

Correspondence: Professor S Perner, MD, PhD, Department of Prostate Cancer Research, Institute of Pathology, University Hospital of Bonn, Sigmund-Freud-Strasse 25, Bonn 53127, Germany.

E-mail: sven.perner1972@gmail.com

${ }^{7}$ The first two authors contributed equally to this work.

Received 14 November 2012; revised and accepted 6 February 2013; published online 26 April 2013 decade. Catalogs of genomic alterations for specific cancer types are leading to a better understanding of cancer biology and promoting the identification of molecular targets for rational therapies. ${ }^{1-3}$ In comparison to conventional chemotherapies, rational therapies have the advantage of a better toxicity profile through target selectivity. ${ }^{4}$ Moreover, they can be as effective as conventional therapies or induce synergistic effects. ${ }^{5-9}$

Recently, we described fibroblast growth factor receptor 1 (FGFR1) as the first actionable target in 
squamous cell lung cancer. FGFR1 is a receptor tyrosine kinase located on chromosome 8p12. Receptor tyrosine kinases commonly contribute significantly to the development of cancer. ${ }^{10}$ When recognizing their fibroblast growth factor ligands, FGFRs undergo dimerization, leading to the phosphorylation of specific intracellular receptor domains, and ultimately leading to the activation of cytoplasmatic effector molecules. The effector pathways following FGFR activation include the Ras/MAPK and PI3/Akt pathways, which in turn transmit the activating signal into the nucleus, where cell differentiation and proliferation are regulated. ${ }^{11}$

FGFR1 amplification was first described as a potential therapeutic target in breast adenocarcinomas. ${ }^{12,13}$ Soon after, Weiss et al $^{14}$ identified FGFR1 amplification as an actionable target in squamous cell carcinoma of the lung. ${ }^{14}$ Of note, FGFR inhibitors in use for clinical trails are not specific to a particular FGFR. The treatment of patients with lung cancer is currently the subject of clinical trials based on FGFR1 amplification, and also based on the fact that both studies mentioned above and also others were able to demonstrate an oncogenic addiction to FGFR1 signaling of FGFR1-amplified cells, which are highly specific to inhibition with pan-FGFR inhibitors, ${ }^{14-16}$ as cells were sensitive to pan-FGFR inhibitors, although FGFR2-4 were not relevantly expressed. ${ }^{17}$ We recently described FGFR1 amplifications in regional lymph node metastases of FGFR1-amplified squamous cell lung cancer, broadening the rationale for therapy with these small-molecule inhibitors to the setting of metastatic disease. ${ }^{18}$

Head and neck squamous cell carcinoma is the most common malignant tumors in the head and neck region. Although some head and neck squamous cell carcinoma can be cured by radical surgical resection, many patients are diagnosed at advanced stages of disease associated with local tumor invasion and metastatic disease. Therefore, the development of effective systemic therapies, including targeted therapies, remains an important area of intense research focus. As was recently the case for squamous cell lung cancer, currently there are no effective targeted therapies available for head and neck squamous cell carcinoma. Given the histomorphological and clinical similarities between head and neck squamous cell carcinoma and squamous cell lung cancer, we proposed that FGFR1 might be involved in the pathogenesis and aggressiveness of head and neck squamous cell carcinoma. Supporting this hypothesis, Freier et $a l^{19}$ recently reported FGFR1 amplifications in $17 \%$ of oral squamous cell carcinomas in a limited number of patient samples. Furthermore, initial functional studies describe a potential role of FGFRs in head and neck squamous cell carcinoma. However, these studies have not examined amplification status of the FGFR1 gene in relation to therapeutical sensitivity. ${ }^{17}$ In this study, we set out to characterize the prevalence of FGFR1 amplification in a comprehensive and clinically wellcharacterized cohort comprising primary, metastatic and recurrent head and neck squamous cell carcinoma of all localizations of the head and neck area.

\section{Materials and methods}

\section{Patient Cohort}

All patients were treated surgically with curative or palliative intent between 1997 and 2011 at the University Hospital of Bonn. Distribution of the clinicopathological data reflects a representative Caucasian cohort of surgically treated head and neck squamous cell carcinoma patients (Supplementary Material 1).

In our study, we included a total of 810 tumor samples derived from 555 patients with head and neck squamous cell carcinoma (488 cases primary tissue available, 23 cases only metastatic tissue available, 14 cases only recurrence tissue available, 5 cases only distant metastases available, 25 cases only clinicopathological data in statistical analysis) (Table 1). Sites of available primary tumor tissue origin were distributed as follows: hypopharynx $(n=54)$, oropharynx $(n=156)$, oral cavity $(n=110)$, larynx $(n=161)$ and unknown in seven patients (carcinoma of unkown primary).

Forty-eight percent (264/555) of all patients presented with lymph node metastasis. In 207/264 cases, we had primary tumor and corresponding lymph node metastasis available, while in 23 patients, we had only metastatic tissue without the corresponding primary tumor, and in 34 cases, lymph node metastases were clinically described, but no tissue was available for assessment. In patients with multiple positive lymph nodes, we assessed up to three lymph node metastases: in 217 cases there was 1 lymph node metastasis available, in 6 cases there was 2 lymph node metastases and in 4 cases there was 3 lymph node metastases, all of which were assessed. Overall, 241 lymph node metastases were analyzed.

Clinically, 24 patients displayed distant metastases (13/24 specimens available). Out of the cases

Table 1 Distribution of our cohort apportioned by tissue availability, assessability and FGFR1 copy number status

\begin{tabular}{|c|c|c|c|c|c|c|}
\hline \multirow[b]{2}{*}{ Tissue } & \multirow[b]{2}{*}{$\begin{array}{l}\text { Tissue } \\
\text { available }\end{array}$} & \multicolumn{2}{|c|}{$\begin{array}{c}\text { Tissue } \\
\text { assessable }\end{array}$} & \multicolumn{3}{|c|}{$\begin{array}{l}\text { FGFR1 copy } \\
\text { number status }\end{array}$} \\
\hline & & Yes & $N o$ & $\begin{array}{c}\text { No } \\
\text { amplification }\end{array}$ & $L L A$ & $H L A$ \\
\hline Primary tumors & 488 & 452 & 36 & 384 & 52 & 16 \\
\hline Metastases & 241 & 223 & 18 & 182 & 32 & 9 \\
\hline Distant metastases & 13 & 13 & 0 & 9 & 3 & 1 \\
\hline Recurrences & 68 & 64 & 4 & 55 & 7 & 2 \\
\hline
\end{tabular}


with an available specimen, 5 cases derived from the lung, 5 cases from soft tissue, 2 cases out of the parotid gland and 1 case of bone metastasis. Out of the 13 available cases with distant metastases, we had corresponding primary head and neck squamous cell carcinoma available in 8 cases. Twentyseven percent $(147 / 555)$ patients experienced recurrence of disease, and recurrent tumor specimens were available in 68 cases. In 54 cases, the corresponding primary and recurrent tumors were both available for analysis. Therapy information, drinking habits and smoking status were available for almost all patients. The study was approved by the institutional ethics review board (no. 148/11).

\section{Tissue Microarray Construction}

Tissue microarray construction was applied as described earlier. ${ }^{20}$ In summary, three representative cores measuring $0.6 \mathrm{~mm}$ in diameter from each formalin-fixed, paraffin-embedded primary tumor, its corresponding lymph node metastasis, distant metastasis, recurrence and benign tissue were assembled into tissue microarray blocks and stained with hematoxylin and eosin (H\&E).

\section{FGFR1 Fluorescence In Situ Hybridization}

Overall, fluorescence in situ hybridization for the detection of FGFR1 amplification status on genomic level was performed as described earlier. ${ }^{14}$ In brief, the FGFR1 target probe (red fluorescent signal) spanning the 8p11.22-23 locus (RP11-148d12) and a commercially available reference probe (green fluorescent signal) located on the centromeric region of chromosome 8 (Metasystems, Altussheim, Germany) were selected for hybridization. Only nuclei displaying green reference signals were included for the determination of the FGFR1 copy number status. All samples were independently analyzed by three evaluators (FG, AF, MB) under a $\times 63$ oil immersion objective with a fluorescence microscope (Zeiss, Jena, Germany). In each case, we assessed at least 100 tumor cell nuclei. A sample was considered amplified if at least $20 \%$ nuclei displayed the FGFR1 amplification. A high-level amplification was defined as additional nine or more red target signals or clusters of target gene signals as compared with the green reference signals. Lower than nine but more than two red target signals as compared with the green reference signals were assigned to be low-level amplified.

\section{P16 Immunohistochemistry}

Serial sections of tissue microarrays were deparaffinized in xylene and stepwise incubated in 100-70\% ethanol. Sections were pretreated in autoclave for
45 min (maximum temperature $125^{\circ} \mathrm{C}$ for $3 \mathrm{~min}$, cool down to $90^{\circ}$ ) and in included pretreatment buffer. Primary monoclonal mouse anti-p16INK4A antibody (CINtec by MTM Laboratories AG, Heidelberg, Germany) was added to the slides for $60 \mathrm{~min}$ at room temperature, followed by incubation with a secondary HRP-conjugated goat anti-mouse antibody (MTM Laboratories) at room temperature for $1 \mathrm{~h}$ (for details see manufacturer's recommendations). After counterstaining of the peroxidase complex, sections were dehydronized and embedded with Eukitt (Fluka).

\section{HPV Analysis}

The HPV Type 3.5 LCD-Array Kit was used for the determination of HPV subtypes by hybridization to $\mathrm{HPV}$-specific DNA probes (Chipron $\mathrm{GmbH}$, Berlin, Germany). Amplification of HPV-specific DNA segments (L1 region) was achieved by using the primer sets HPV '125' and HPV MY09/MY11. Ten microliters of the amplification products were hybridized to HPV type-specific capture probes fixed to an LCD array chip. All steps were performed according to the manufacturer's recommendations.

\section{Statistical Analysis}

All statistical analysis and graphical output were carried out with $\mathrm{R}$ version 2.13.0.

For ordinally scaled nonparametric data, the Wilcoxon-Mann-Whitney $U$-test $(\mathrm{W}-\mathrm{M}-\mathrm{W}-U$ ) was used. In case of more than two groups, we used the extended $\mathrm{W}-\mathrm{M}-\mathrm{W}-U$ test. Fisher's exact test was used for computing statistical significance. Mean value comparison were carried out by $t$-test or analysis of variance in case of more groups than two. In all tests, 0.05 was chosen as the level of significance. Two groups according to the median value were defined for age (62 years), recurrencefree survival (13 months) and overall survival (26 months). In Figure 2, values are displayed in logarithmic manner for better visualization. As the number 1 becomes 0 , we added 1 count to every value to enable visualization of small numbers.

\section{Results}

\section{FGFR1 Amplification Status in Primary Head and Neck Squamous Cell Carcinoma}

Of the 488 analyzed primary tumor samples, 93\% (452/488) cases were assessable. Of these, 23\% (103/ $452)$ were from the oral cavity, 33\% (148/452) from the oropharynx, $10 \%(47 / 452)$ from the hypopharynx and $33 \%(147 / 452)$ from the larynx, whereas in $7(2 \%)$ cases the primary site was unknown. Of all assessable primary tumors, $15 \%(68 / 452)$ displayed an FGFR1 amplification with $12 \%(52 / 452)$ lowlevel and 4\% high-level amplification (16/452). 
FGFR1 amplification was most common in primary squamous cell carcinomas of the hypopharynx $(23 \% ; 11 / 47)$ and larynx $(18 \% ; 26 / 147)$, and less common in squamous cell carcinomas of the oropharynx $(14 \% ; 21 / 148)$ and oral cavity $(9 \% ; 9 /$ 103), which are the areas commonly afflicted by HPV infection $(P<0.05)$. Control samples of benign squamous cell tissue were always negative for FGFR1 amplification.

\section{FGFR1 Amplification Status in Metastatic Head and Neck Squamous Cell Carcinoma}

Of 264 patients with clinically determined metastatic diseases, we successfully assessed 223 lymph node metastases. Eighteen percent (41/223) displayed FGFR1 amplification with 14\% low-level amplification (32/223) and 4\% high-level amplification (9/223).

Of the 207 cases with available corresponding primary tumor and lymph node metastasis, we had 26 non-comparable cases and 181 cases in which the primary and corresponding lymph node metastasis tissues were both assessable. Eighty-eight percent
(160/181) of cases displayed a concordant FGFR1 copy number status in the primary and metastatic tissue. Of these, we found $4 \%(6 / 160)$ of the cases to be high-level amplified (Figure 1a and a1), 8\% (13/160) cases low-level amplified and 88\% (141/160) of the cases displayed no amplification (Figure 1c and c1). In 12\% (21/181) cases, FGFR1 copy number status was discordant (Figure 2a).

In the seven cases with more than one lymph node metastasis, all cases were assessable. Four cases had the same copy number status in all analyzed tissue and three cases had differing FGFR1 copy numbers.

Furthermore, we had tumor tissue of 13 distant metastases. Of these, four displayed FGFR1 amplification (2/4 lung, 2/4 others) and nine wild-type FGFR1 (Figure 1c1) (3/9 lung, 6/9 others). Out of the eight corresponding cases of primary head and neck squamous cell carcinoma and distant metastases, five cases had identical FGFR1 copy number status ( $3 \times$ lung, $2 \times$ others), all of which displayed a wildtype FGFR1 copy number status. In three cases, we had differing FGFR1 copy number status in primary tumor and distant metastases $(2 \times$ lung, $1 \times$ others $)$ (Figure 2b).
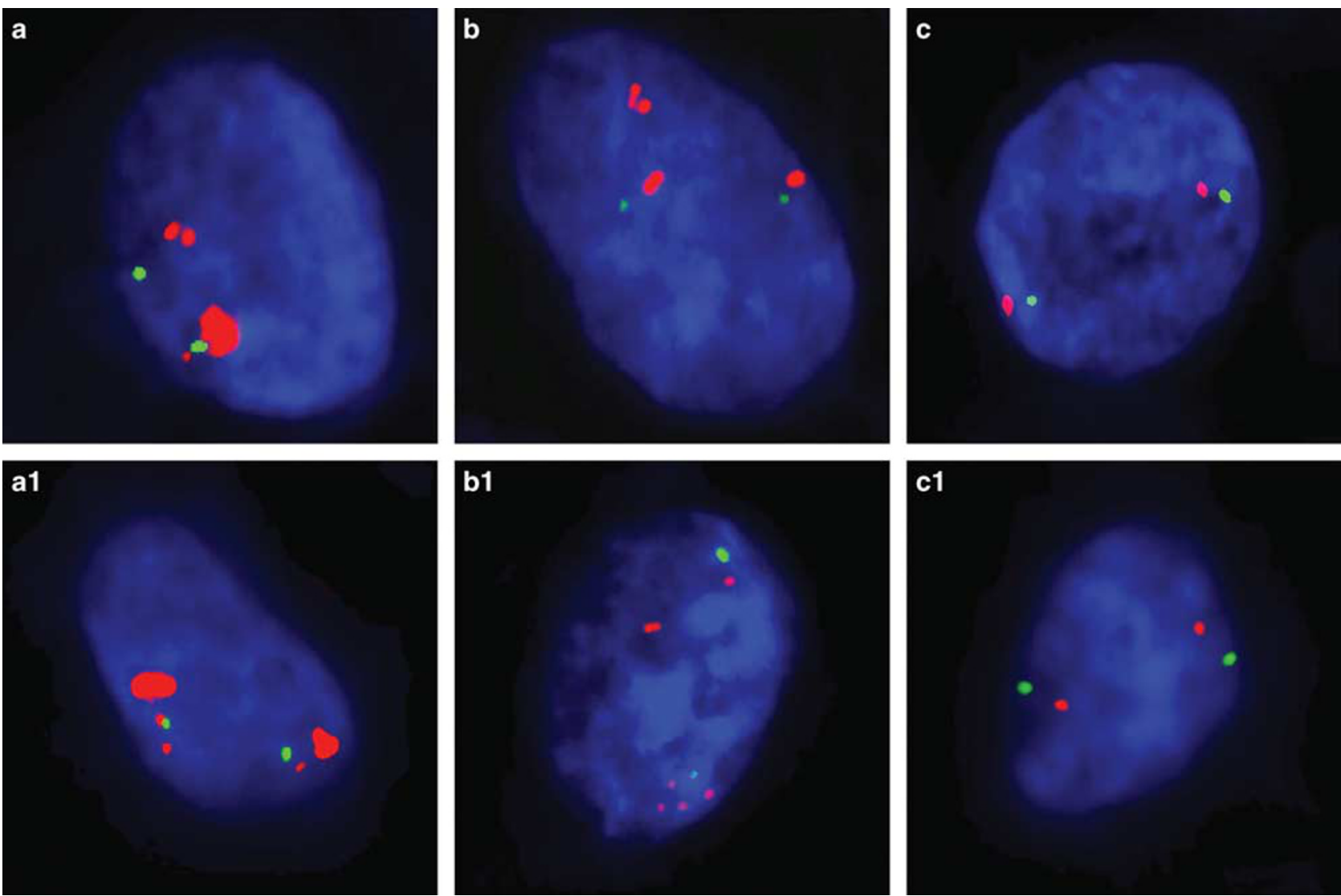

Figure 1 FISH images of primary tumors (a, b, c) (upper row) and their corresponding tumor tissues (lower row) (a1, b1, c1). A high level amplification (HLA) is represented by a primary tumor (a) and its corresponding lymph node metastasis (a1). (b) is an example of low level amplification (LLA) in a primary tumor and its corresponding recurrent tumor tissue (b1). An example of a wild type copy status is shown in a primary tumor (c) and its corresponding distant metastasis (c1). 

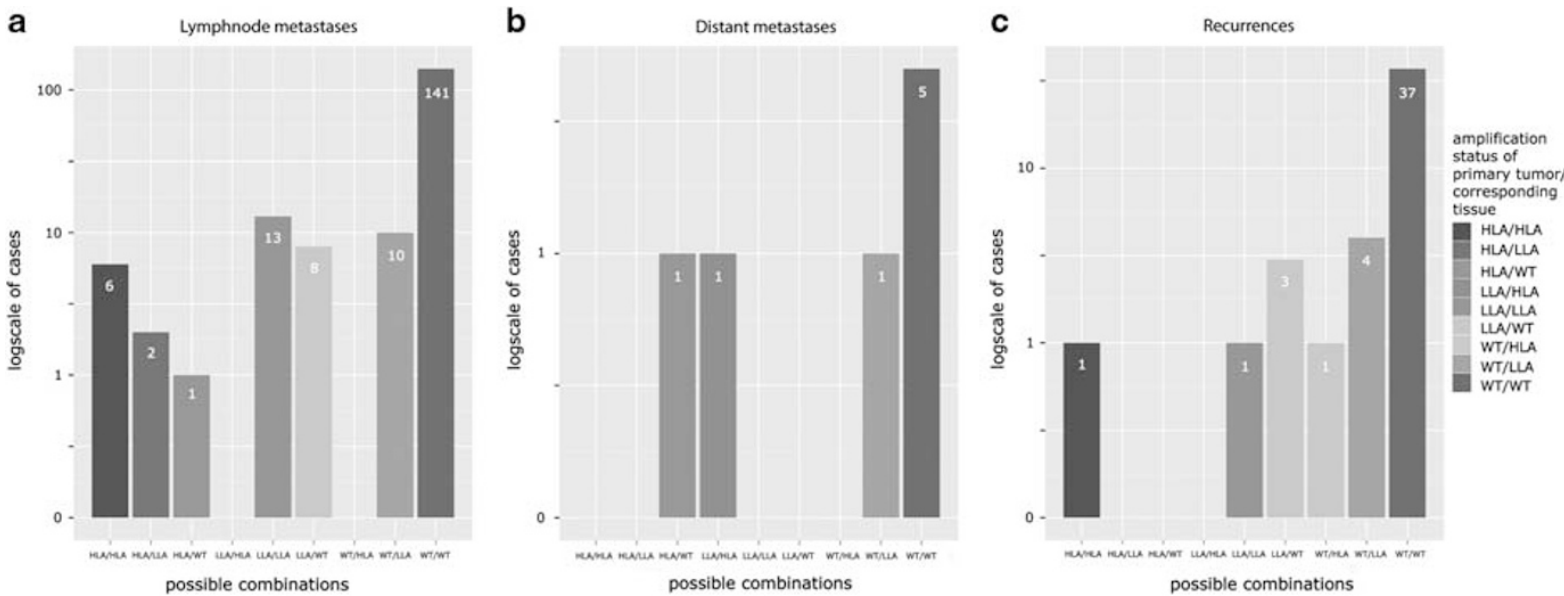

Figure 2 Histogram of FGFR1 copy number status and corresponding tumor tissue. (a) Histogram of FGFR1 amplification status in primary tumors and corresponding lymph-node metastases. The colors indicate the possible combinations of different amplification levels. (b) Histogram of the corresponding FGFR1 amplification status in primary tumor and its corresponding distant metastasis. (c) Histogram of the corresponding FGFR1 amplification status in primary tumor and its corresponding recurrent tumor. Data was logarithmically plotted. In order to include all detected cases, the value "1" was added to every count (for detailed information, see description statistical analysis in materials and methods).

\section{FGFR1 Amplification in Recurrent Head and Neck Squamous Cell Carcinoma}

A total of 68 cases of recurrent tumor tissue were available. Of these, $94 \%(64 / 68)$ were assessable. Primary and recurrent tumor tissue of the same patient were available in 54 cases, of which 47 (87\%) were assessable: 83\% (39/47) presented an identical copy number status in both primary tumor and recurrent tumor tissue (example of concordant wild-type FGFR1; Figure 1b and b1). In eight cases, the recurrent tumor displayed a different FGFR1 copy number status as compared with the primary tumor (Figure 2c).

\section{FGFR1 Amplification and Clinicopathological} Features of Head and Neck Squamous Cell Carcinoma

All features of clinicopathological data in relation to FGFR1 copy number status and statistical analysis are summarized in Table 2.

In detail, parameters of age at primary diagnosis and gender were available for all patients. FGFR1 amplification was not significantly associated with age $(P=0.30)$, but occurred significantly more often in male patients $(P=0.02)$.

Smoking status was available for 434 patients. Of these, 352 had FGFR1 assessable tumor tissue available. Eighty-eight percent (310/352) of assessable patients were smokers and $12 \%(42 / 352)$ were never-smokers. FGFR1 amplification occurs significantly more often in the cohort of patients with a smoking history than in the group of patients who never smoked $(P=0.04)$. We also compared the number of pack years with the occurrence of FGFR1 amplifications. Interestingly, FGFR1 amplifications occur significantly more often with rising amount of pack years $(P=0.02)$. Status of alcohol abuse was available for 411 patients, out of which 331 had FGFR1 assessable tumor tissue available. Among the patients, 130 were never-drinkers, 73 were occasional drinkers and 128 medium to heavy drinkers. FGFR1 amplification occurs significantly more often with rising alcohol consumption $(P<0.05)$.

Furthermore, we assessed the association between FGFR1 amplification status and pathologic features of primary tumors. We grouped lower (pT1 and pT2) and higher T-stages (pT3 and pT4) and assessed if FGFR1 amplification correlated with these subgroups. We found a significant association of FGFR1 amplifications and higher T-stages $(P<0.05)$. Also, FGFR1 amplification was significantly associated with the presence of lymphovascular invasion (L) $(P=0.02)$.

To assess whether FGFR1 amplification status predicts occurrence of regional lymph node metastases, we correlated the FGFR1 amplification status of primary tumors with clinical nodal status. Of the cases with FGFR1 amplification $(n=68)$ in the primary tumor, $40 \%(27 / 68)$ had lymph nodenegative disease and $60 \%(41 / 68)$ had lymph nodepositive disease compared with 61\% (209/384) lymph node-negative and 46\% (175/384) lymph node-positive of cases without FGFR1 amplification $(n=384)$, indicating that FGFR1 amplification is significantly associated with the development of lymph node metastasis $(P=0.02)$.

Patients were screened for HPV infection by p16 immunohistochemistry staining of all patients' tissue, which revealed a positive immunohistochemistry status in 10\% (47/488) of the cases. These cases were then investigated by LCD array, which further identified the occurrence and the subtypes of HPV. Seventy-seven percent (36/47) of p16-positive cases turned out to be truly HPV positive, resulting in an overall frequency of $7 \%(36 / 488)$ within our cohort. We found HPV16 in 32 cases, the combination 
Table 2 Summary of clinico-pathological data in relation to FGFR1 amplification

\begin{tabular}{|c|c|c|c|c|c|}
\hline & $\begin{array}{c}\text { No } \\
\text { amplification }\end{array}$ & $\begin{array}{l}\text { Low-level } \\
\text { amplificationn }\end{array}$ & $\begin{array}{l}\text { High-level } \\
\text { amplification }\end{array}$ & $\begin{array}{c}\text { Any } \\
\text { amplification }\end{array}$ & $\begin{array}{c}\text { P-value } \\
\text { (Fisher's exact test) }\end{array}$ \\
\hline \multicolumn{6}{|l|}{ Age (years), median } \\
\hline$<62$ & 185 & 22 & 6 & 28 & 0.295 \\
\hline$\geq 62$ & 199 & 30 & 10 & 40 & \\
\hline \multicolumn{6}{|l|}{ Gender } \\
\hline Female & 96 & 6 & 2 & 8 & $0.017^{*}$ \\
\hline Male & 288 & 46 & 14 & 60 & \\
\hline \multicolumn{6}{|l|}{ Site of origin } \\
\hline Oral, oropharynx & 221 & 21 & 9 & 30 & $0.045^{*}$ \\
\hline Hypopharynx, larynx & 157 & 30 & 7 & 37 & \\
\hline Unknown & 6 & 1 & 0 & 1 & \\
\hline \multicolumn{6}{|l|}{ Tobacco } \\
\hline Never-smoker & 40 & 1 & 1 & 2 & $0.04153^{*}$ \\
\hline Smoker & 255 & 43 & 12 & 55 & \\
\hline \multicolumn{6}{|l|}{ Alcohol } \\
\hline Non-drinker & 119 & 9 & 2 & 11 & $0.007795 * *$ \\
\hline Occasional & 60 & 10 & 3 & 13 & \\
\hline Medium-heavy & 100 & 21 & 7 & 28 & \\
\hline \multicolumn{6}{|l|}{ Pathological features } \\
\hline T1-T2 & 239 & 17 & 5 & 22 & $0.000 * * *$ \\
\hline $\mathrm{T} 3-\mathrm{T} 4$ & 123 & 31 & 10 & 41 & \\
\hline \multicolumn{6}{|c|}{ Lymphovascular invasion } \\
\hline Negative & 336 & 44 & 11 & 55 & $0.02 *$ \\
\hline Positive & 29 & 7 & 4 & 11 & \\
\hline \multicolumn{6}{|c|}{ Lymph node metastases (at the time of presentation) } \\
\hline Negative & 182 & 18 & 4 & 22 & $0.02 *$ \\
\hline Positive & 175 & 30 & 11 & 41 & \\
\hline \multicolumn{6}{|c|}{ Distant metastases (at the time of presentation) } \\
\hline Negative & 375 & 48 & 14 & 62 & $0.015^{*}$ \\
\hline Positive & 9 & 4 & 2 & 6 & \\
\hline \multicolumn{6}{|l|}{ HPV status } \\
\hline Negative & 351 & 52 & 16 & 68 & $0.003127 * *$ \\
\hline Positive & 36 & 0 & 0 & 0 & \\
\hline \multicolumn{6}{|c|}{ Survival } \\
\hline \multicolumn{6}{|c|}{ Recurrence-free survival, median 28 months } \\
\hline$<28$ & 107 & 14 & 8 & 22 & 0.6116 \\
\hline \multirow{2}{*}{\multicolumn{6}{|c|}{ Overall survival, median 26 months }} \\
\hline & & & & & \\
\hline$<26$ & 177 & 25 & 10 & 35 & 0.5959 \\
\hline$\geq 26$ & 190 & 27 & 5 & 32 & \\
\hline
\end{tabular}

Features include age, gender, site of origin, tobacco, alcohol, pathologic features, HPV status treatment regimen and survival.

$P$-values indicate the significance of any FGFR1 amplification (HLA and LLA vs. non-amplified) between the described subgroups and are calculated by Fisher's exact test.

Significance levels are $0.05(*), 0.01(* *)$ and $0.001(* * *)$.

of HPV16 and HPV35 one time, HPV35 one time and HPV33 two times. All cases displaying HPV infection had a wild-type FGFR1 status. All FGFR1-amplified tumors lacked HPV infection. HPV-positive patients had a significantly better overall survival compared with HPV-negative patients $(P<0.05)$.

\section{Treatment and Outcome}

After primary surgical treatment of localized disease, 147 patients suffered from a recurrence, 87 local, 49 as lymph node metastases and 28 as distant metastases. The median time until recurrence was 13 months, which did not differ among tumors on the basis of FGFR1 amplification status $(P=0.9)$. Of these 147 patients, we had assessable primary and recurrent tumor tissue of 47 patients available. Seventeen patients underwent surgical treatment only, 15 patients were treated with postsurgical radiation, 10 patients with postsurgical radiochemotherapy and 3 patients were treated with radiochemotherapy only. In two cases, there was no therapy regimen determinable. Comparing FGFR1 copy number status of primary tumors and recurrences, we found 39 patients with concordant and 


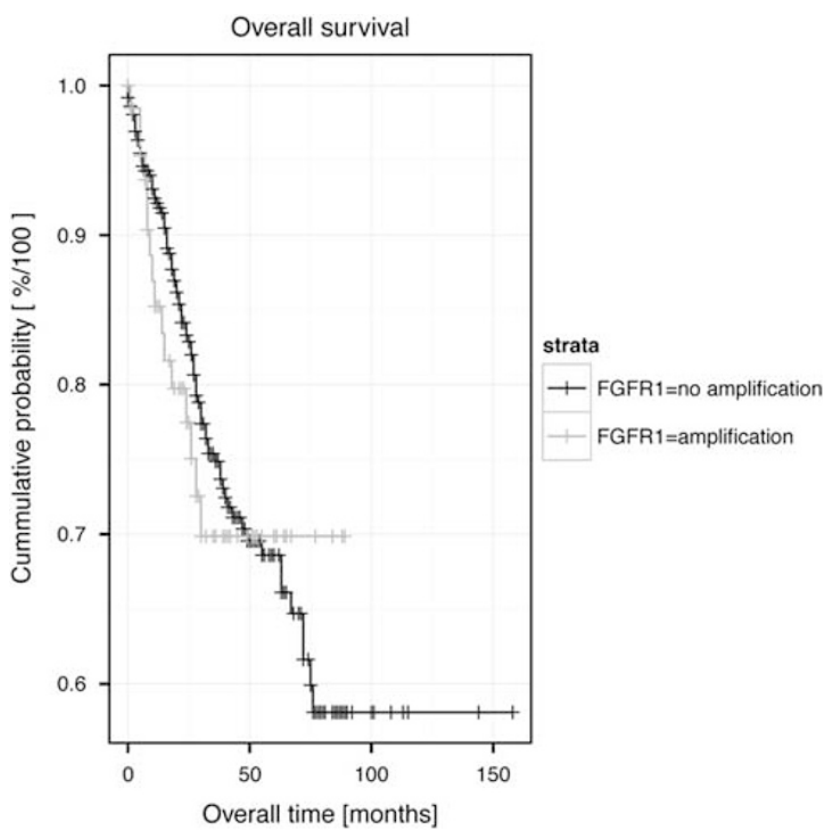

Figure 3 Kaplan Meier curve for overall survival related to FGFR1 copy number status. FGFR1 amplification is not significantly associated with better or worse overall survival.

8 patients with discordant gene copy number status in these tissues (for details see above). As discordance only occurred in eight patients, who underwent different therapy regiments (ie $4 \times$ surgery, $2 \times$ surgery and adjuvant radiochemotherapy, $1 \times$ surgery and radiation and $1 \times$ radio-chemotherapy), we do not observe a direct influence of a specific therapy regimen on the change of FGFR1 copy number status from primary tumor to recurrent tumor tissue. Patient number of discordant FGFR1 status is also underpowered for a reliable statistical analysis. There is no significant difference in overall survival between patients harboring a FGFR1 amplification and those with FGFR1 wild type $(P=0.71)$ (Figure 3$)$.

\section{Discussion}

Frequent genetic alterations associated with tumorigenesis in cancers have become a major focus of research in hopes that such alterations will represent opportunities for the development of targeted therapies. ${ }^{1}$ So far, patients with head and neck squamous cell carcinoma lack targeted therapy options and still have a rather limited functional and poor overall outcome in spite of improvements in conventional treatment modalities such as surgery and radiochemotherapy.

We and others previously discovered the highly smoking-related FGFR1 amplification in approximately a fifth of all primary squamous cell lung cancer. ${ }^{14,15}$ This discovery resulted in the immediate initiation of a phase I clinical trial of small-molecule FGFR inhibitor therapy that is enrolling patients diagnosed with stage IV disease suffering from the second recurrence with FGFR1amplified primary squamous cell lung cancer.

Owing to known similarities in etiology, histology and risk factors between head and neck squamous cell carcinoma and squamous cell lung cancer, we hypothesized that FGFR1 amplifications are also involved in the pathogenesis of head and neck squamous cell carcinoma. To test this hypothesis, we examined a large number of primary and metastatic head and neck squamous cell carcinoma to determine the prevalence of FGFR1 amplification in these tumors. We found an overall FGFR1 amplification rate of $15 \%$ in primary head and neck squamous cell carcinoma, which is close to the frequency described previously by Weiss et $a I^{14}$ for squamous cell lung cancer and also by Freier et al ${ }^{19}$ for oral squamous cell carcinomas. Mirroring our findings in lung cancers, ${ }^{14}$ acquisition of FGFR1 amplification in head and neck squamous cell carcinoma showed a dose-dependent association with exposure to chemical carcinogens (eg smoking and, in the case of head and neck squamous cell carcinoma, alcohol abuse). In contrast, no FGFR1 amplification was found in the prognostically more benign HPV-driven tumors, reinforcing the concept that this tumor subtype has its own specific pathogenesis and behavior. Confirming these observations, FGFR1 amplification was detected more frequently in the squamous cell carcinoma of the hypopharynx and larynx, which are rather driven by chemical carcinogens than by HPV, as opposed to squamous cell carcinoma of the oropharynx and oral cavity, which are HPV positive in $30-65 \%$ of cases. $^{21,22}$ Interestingly, positive HPV status and FGFR1 amplification occurs mutually exclusively, implying completely independent pathways of carcinogenesis. This raises interesting questions regarding whether well-characterized downstream effectors of HPV infection that lead to head and neck squamous cell carcinoma are also involved in tumors characterized by FGFR1 amplification. Smoking, on the other hand, was clearly associated with FGFR1 amplification, raising interesting questions for future studies involving the mechanistic relationship between tobacco-associated cell damage and FGFR1 amplification. Likewise, FGFR1 amplification was seen more commonly among patients consuming alcohol, again providing fertile ground for new insights into the pathogenesis of head and neck squamous cell carcinoma. Damage occurring to the tissue because of radiation and/or chemotherapy does not seem to have an influence on the frequency of FGFR1 amplification, as recurrence of tumors treated under these initially employed therapy regimens do not harbor more FGFR1 amplifications than tumors treated purely with surgical resection.

As FGFR1 is on its way to becoming an actionable target, it is important to consider patients with 
multifocal disease, to assess whether separate tumor sites in a single patient should be tested individually for FGFR1 amplification status. To that end, we assessed a large number of cases with corresponding lymph node metastases, and a number of cases with corresponding distant metastases, as well as corresponding recurrent tumors. We found that the FGFR1 gene status is concordant in the vast majority of patients with metastatic or recurrent disease, although we did find exceptions to this tendency. Tissue microarrays were constructed by random selection of tissue cores from the whole tumor. Therefore, discordance could be due to molecular heterogeneity within the whole tumor. However, in fact, we assessed whole tumor sections in a subset of discordant cases and found that the distribution of FGFR1 copy number status is the same as assessed on the tissue microarrays. Consequently, results derived from the tissue microarrays are reliable, although a theoretical limitation with regard to a sampling bias remains.

However, the clinical significance of this discordance is subject to further studies. In the meantime, it appears advisable to test all available tissue sites for FGFR1 amplification status in patients being considered for treatment with FGFR inhibitors.

We assessed the utility of FGFR1 amplification status as a prognostic biomarker, and although we did not find differences in survival time on the basis of FGFR1 amplification, we did demonstrate an association between FGFR1 amplification and various well-characterized parameters of poor prognosis, including advanced T-stage, lymphovascular invasion and regional and distant metastatic disease. As HPV infection is commonly linked with a favorable prognosis, ${ }^{23}$ our data associating FGFR1 amplification with a worse prognosis is consistent with mutual exclusion of HPV infection and FGFR1 amplification. The lack of correlation between FGFR1 amplification and overall survival could be due to the pronounced heterogeneity of our cohort with respect to treatment regimens.

In summary, FGFR1 amplification is a frequent event in HPV-negative head and neck squamous cell carcinoma, and FGFR1 copy number status was concordant between primary and metastatic tumors in the vast majority of cases. In the era of personalized cancer treatment, our findings emphasize the need to establish the prognostic and predictive significance of molecular alterations like FGFR1 amplification for head and neck squamous cell carcinoma and to incorporate them into clinical algorithms when rational specific therapies exist. Further research will be necessary to elucidate the mechanistic relationships between FGFR1 amplification and other well-established risk factors for head and neck squamous cell carcinoma, such as tobacco and alcohol, and molecular differences to HPV infection-driven head and neck squamous cell carcinoma.

\section{Acknowledgements}

We thank Mareike Tonndorf for her assistance in HPV analysis. We also thank Susanne Steiner for assistance of p16 staining. This study was supported by a grant of the Rudolph-Becker-Foundation (to SP), by a BONFOR grant of the Medical Faculty of the University of Bonn (to MB) and a grant of the DFG (to SP (no. PE 1179/4-1) and CL (no. LE 2483/4-1)).

\section{Disclosure/conflict of interest}

The authors declare no conflict of interest.

\section{References}

1 Beroukhim R, Mermel CH, Porter D, et al. The landscape of somatic copy-number alteration across human cancers. Nature 2010;463:899-905.

2 Frohling S, Dohner H. Chromosomal abnormalities in cancer. N Engl J Med 2008;359:722-734.

3 Weir B, Zhao X, Meyerson M. Somatic alterations in the human cancer genome. Cancer Cell 2004;6: 433-438.

4 Bearz A, Berretta M, Lleshi A, et al. Target therapies in lung cancer. J Biomed Biotechnol 2011;2011:921231.

5 Reck M, von Pawel J, Zatloukal P, et al. Phase III trial of cisplatin plus gemcitabine with either placebo or bevacizumab as first-line therapy for nonsquamous non-small-cell lung cancer: AVAil. J Clin Oncol 2009;27:1227-1234.

6 Sandler A, Gray R, Perry MC, et al. Paclitaxelcarboplatin alone or with bevacizumab for non-smallcell lung cancer. N Engl J Med 2006;355:2542-2550.

7 Mitsudomi T, Morita S, Yatabe Y, et al. Gefitinib versus cisplatin plus docetaxel in patients with non-smallcell lung cancer harbouring mutations of the epidermal growth factor receptor (WJTOG3405): an open label, randomised phase 3 trial. Lancet Oncol 2010;11: 121-128.

8 Pirker R, Pereira JR, Szczesna A, et al. Cetuximab plus chemotherapy in patients with advanced non-smallcell lung cancer (FLEX): an open-label randomised phase III trial. Lancet 2009;373:1525-1531.

9 Wagner PL, Perner S, Rickman DS, et al. In situ evidence of KRAS amplification and association with increased p21 levels in non-small cell lung carcinoma. Am J Clin Pathol 2009;132:500-505.

10 Wesche J, Haglund K, Haugsten EM. Fibroblast growth factors and their receptors in cancer. Biochem J 2011;437:199-213.

11 Powers CJ, McLeskey SW, Wellstein A. Fibroblast growth factors, their receptors and signaling. Endocr Relat Cancer 2000;7:165-197.

12 Turner N, Grose R. Fibroblast growth factor signalling: from development to cancer. Nat Rev Cancer 2010;10:116-129.

13 Turner N, Pearson A, Sharpe R, et al. FGFR1 amplification drives endocrine therapy resistance and is a therapeutic target in breast cancer. Cancer Res 2010;70:2085-2094.

14 Weiss J, Sos ML, Seidel D, et al. Frequent and focal FGFR1 amplification associates with therapeutically 
tractable FGFR1 dependency in squamous cell lung cancer. Sci Transl Med 2010;2:1-7.

15 Dutt A, Ramos AH, Hammerman PS, et al. Inhibitorsensitive FGFR1 amplification in human non-small cell lung cancer. PLoS One 2011;6:e20351.

16 Reis-Filho JS, Simpson PT, Turner NC, et al. FGFR1 emerges as a potential therapeutic target for lobular breast carcinomas. Clin Cancer Res 2006;12:6652-6662.

17 Marshall ME, Hinz TK, Kono SA, et al. Fibroblast growth factor receptors are components of autocrine signaling networks in head and neck squamous cell carcinoma cells. Clin Cancer Res 2011;17:5016-5025.

18 Goeke F, Franzen A, Menon R, et al. Rationale for treatment of metastatic squamous cell carcinoma of the lung using FGFR Inhibitors. Chest 2012;142: 1020-1026.

19 Freier K, Schwaenen C, Sticht C, et al. Recurrent FGFR1 amplification and high FGFR1 protein expression in oral squamous cell carcinoma (OSCC). Oral Oncol 2007;43:60-66.

20 Perner S, Wagner PL, Soltermann A, et al. TTF1 expression in non-small cell lung carcinoma: association with TTF1 gene amplification and improved survival. J Pathol 2009;217:65-72.

21 Syrjanen K, Vayrynen M, Castren O, et al. Morphological and immunohistochemical evidence of human papilloma virus (HPV) involvement in the dysplastic lesions of the uterine cervix. Int J Gynaecol Obstet 1983;21:261-269.

22 Chaturvedi AK, Engels EA, Anderson WF, et al. Incidence trends for human papillomavirus-related and -unrelated oral squamous cell carcinomas in the United States. J Clin Oncol 2008;26:612-619.

23 Schlecht NF. Prognostic value of human papillomavirus in the survival of head and neck cancer patients: an overview of the evidence. Oncol Rep 2005;14:1239-1247.

Supplementary Information accompanies the paper on Modern Pathology website (http://www.nature.com/ modpathol) 